TITLE:

\title{
Measurement and Visualization of IIIF Image Usages.
}

AUTHOR(S):

Nishioka, Chifumi; Nagasaki, Kiyonori

\section{CITATION:}

Nishioka, Chifumi ...[et al]. Measurement and Visualization of IIIF Image Usages.. Digital Libraries at the Crossroads of Digital Information for the Future 2019: 308-311

\section{ISSUE DATE:}

2019

URL:

http://hdl.handle.net/2433/244866

\section{RIGHT:}

This is a post-peer-review, pre-copyedit version of an article published in Digital Libraries at the Crossroads of Digital Information for the Future. The final authenticated version is available online at: http://dx.doi.org/10.1007/978-3-03034058-2_30:; The full-text file will be made open to the public on 29 October 2020 in accordance with publisher's 'Terms and Conditions for Self-Archiving'.; この論文は出版社版でありません。引用の際には出版社版をご確認ご利用ください

。; This is not the published version. Please cite only the published version. 


\title{
Measurement and Visualization of IIIF Image Usages
}

\author{
Chifumi Nishioka ${ }^{1[0000-0002-1853-3038]}$ and Kiyonori \\ Nagasaki ${ }^{2}[0000-0002-5485-0567]$ \\ 1 Kyoto University Library, Kyoto, Japan \\ nishioka.chifumi.2c@kyoto-u.ac.jp \\ 2 The University of Tokyo, Tokyo, Japan \\ nagasaki@dhii.jp
}

\begin{abstract}
In these years, a lot of libraries and museums have adopted IIIF (International Image Interoperability Framework), which promotes mutual use of images among institutions. In IIIF-compatible digital collections, images are retrieved via IIIF Image API with specifying regions of images. Thus, we can investigate the detailed image usage by examining requested regions. In this paper, we demonstrate an application that measures and visualizes which regions of images are looked by users. Specifically, we count the number of accesses to each pixel of images. Since a pixel is the smallest unit that composes an image, it enables to show the detailed image usage. Finally, we visualize the result as heat maps and display heat maps over original images on Mirador, a IIIFcompatible image viewer. Thus, users can interactively investigate which regions of images are looked by users with panning and zooming.
\end{abstract}

Keywords: Visualization · Usage statistics · Digital Humanities · Image.

\section{Introduction}

It is important for libraries and museums to understand how digital collections and their contents have been used for many reasons such as improvement of digital collections [2] and accountability for stakeholders. The usage has been analyzed following two steps as below:

1. Selection of a metric. A metric that suits for the purpose of the analysis is chosen. Then, the metric is measured based on data such as server logs and loan records. So far, metrics such as the number of accesses to materials (e.g., historical manuscripts) and images have been widely employed [3].

2. Visualization of the result. The result of the analysis is visualized to facilitate users to understand. For instance, bar charts and line chars have been used.

In these years, a lot of digital collections from libraries and museums have adopted IIIF (International Image Interoperability Framework) [5], which promotes mutual use of images among institutions. IIIF defines a set of APIs to 
enable interoperable use of images over different institutions. In IIIF-compatible digital collections, images are fetched via IIIF Image $\mathrm{API}^{3}$, whose syntax is defined as: $\{$ scheme $\}: / /$ server\} $/$ prefix $\} /\{$ identifier $\} /$ region\} $/$ size $/$ \{rotation\}/\{quality\}. \{format\}. Every time an image is zoomed and panned on an image viewer, the image is called via IIIF Image APIs with varying the value of region. Therefore, we can analyze the detailed image usage by examining the values of region. In this paper, we demonstrate how to measure the detailed image usage and how to visualize the result of the analysis. Specifically, we employ the number of accesses to each pixel as a metric. Since a pixel is the smallest unit that composes an image, we can see the detailed image usage. Finally, we visualize the result of the analysis by heat maps. Heat maps are shown over original images using Mirador ${ }^{4}$, an IIIF-compatible image viewer, which has a function of panning and zooming images. Thus, users (e.g., administrator of a digital collection) can interactively see which regions of images have been looked.

\section{Approach to Measure and Visualize IIIF Image Usage}

Below, we describe how to measure the number of accesses to each pixel, how to generate heat maps, and how to display heat maps for users in each paragraph.

Measure the number of accesses to each pixel. For each image, an $H \times W$ matrix, where all elements are 0 , is generated. $H$ and $W$ are the height and width of the image in pixel, respectively. Thus, each element of the matrix corresponds to each pixel of the image. The height and width of images are programatically retrieved by info.json ${ }^{5}$ provided by the IIIF Image API. Subsequently, the requested images and regions of each image are acquired by parsing server logs of IIIF Image API. Based on the requested regions, the number of accesses to each pixel of each image is counted and recorded to the matrices.

Generation of heat maps After counting the number of accesses to each pixel, the result is outputted as a heat map. The RGB value of each pixel is calculated considering the minimum and maximum values of the number of accesses to pixel in an image.

Display of heat maps Generated heat maps are displayed over corresponding target images, in order to enable users (e.g., administrator) to understand the detailed image usage. The IIIF Presentation API enables to overlay images by specifying to display the two images on a page. Mirador, one of the most popular image viewers among the IIIF community, implements a function of overlay display of images as shown in Figure 1. One can manipulate the visibility and opacity for each image in the left side panel and interactively see which regions of images are looked by users with panning and zooming.

\footnotetext{
${ }^{3}$ https://iiif.io/api/image/

${ }^{4}$ https://projectmirador.org/

${ }^{5}$ https://iif.io/api/image/2.1/\#image-information-request-uri-syntax
} 


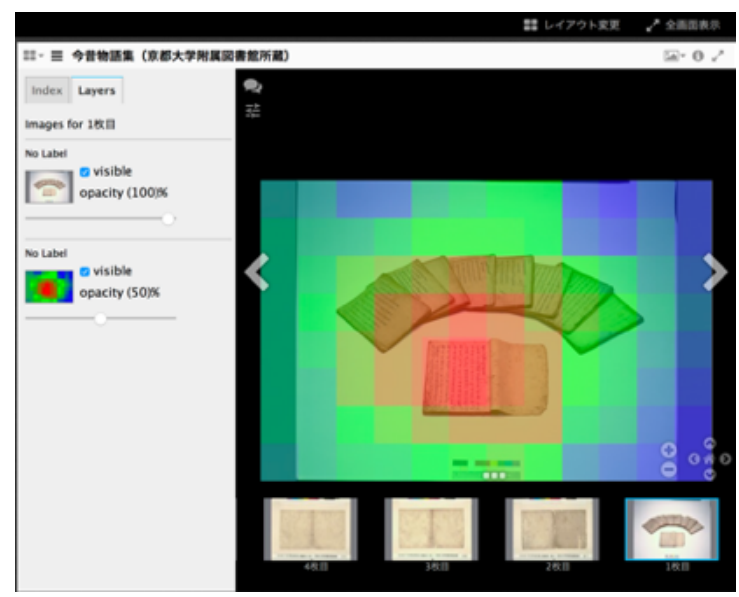

Fig. 1: Overlay display of a heat map and its target image using Mirador. Photograph courtesy of the Main Library, Kyoto University - Konjaku monogatarishuu.

We show two examples of the result of the analysis of the image usage in below. In Figure 2a, we observe a slight tendency that accesses are made along with the Japanese archipelago. As shown in Figure 2b, we observe images with concentrated accesses in specific regions. Referrers of server logs reveal that these regions are referenced from an external platform. For the future work, indication of the referrer might enable to show the motivation and background behind accesses to users.

\section{Possible Applications}

This section lists possible applications of the analysis of the image usage.

Collaborative research platform The data model used in IIIF follows the Web Annotation Data Model ${ }^{6}$. Thus, IIIF facilitates to share not only images but also information accompanying images (e.g., annotations such as transcriptions). For this reason, IIIF-compatible collaborative research platforms have been developed [4]. Heat maps enable researchers to understand regions of images already examined by collaborators.

Transcription Platform In the decades, a lot of transcription projects and platforms have been launched [1]. Transcribers zoom and pan images during generating transcriptions. If a platform is compatible with IIIF, it is possible to verify a pattern such as whether there is a difference in transcription performance (e.g., accuracy) between regions being zoomed and those being not zoomed. If we find a pattern, we can facilitate verification process for transcriptions.

\footnotetext{
${ }^{6}$ https://www.w3.org/TR/annotation-model/
} 


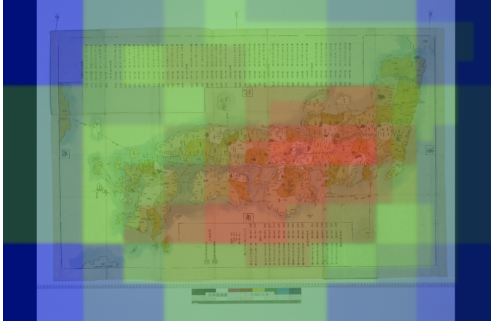

(a) An image where we observe a slight tendency that accesses are made along the Japanese archipelago. Photograph courtesy of the Main Library, Kyoto University - Nihonkokubizu.

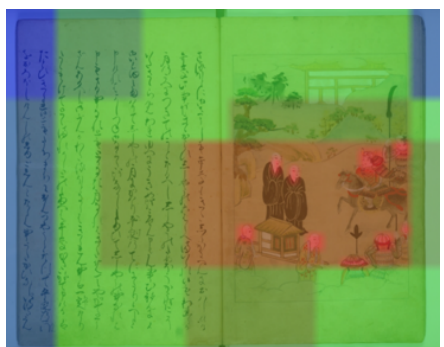

(b) An image with concentrated accesses in specific regions. Photograph courtesy of the Main Library, Kyoto University - The story of Benkei, a tragic warrior.

Fig. 2: Examples of the result of the analysis of the image usage

\section{Conclusion}

In this paper, we show a demonstration, where the detailed image usage are measured and visualized for IIIF-compatible digital collections. Specifically, we count the number of accesses to each pixel of images. Thus, it enables the finegrained analysis of image usage, because a pixel is the smallest unit of an image. Finally, we visualize the result as heat maps and show heat maps over target images using Mirador. Therefore, users can interactively investigate which regions of images are looked with panning and zooming.

\section{References}

1. Carletti, L., Giannachi, G., Price, D., McAuley, D., Benford, S.: Digital humanities and crowdsourcing: An exploration. In: Museums and the Web. Museums and the Web (2013)

2. Hughes, L.: Evaluating and Measuring the Value, Use and Impact of Digital Collections. Facet (2011). https://doi.org/10.29085/9781856049085

3. Jones, S., Cunningham, S.J., McNab, R., Boddie, S.: A transaction log analysis of a digital library. International Journal on Digital Libraries 3(2), 152-169 (2000)

4. Sato, M., Ota, I.: Collaboration system based on crowdsourcing with mirador proposal of a system to support analysis and theory in collaborative research of humanities. IPSJ SIG-CH Technical Report 2017-CH-114(7), 1-6 (2017)

5. Snydman, S., Sanderson, R., Cramer, T.: The international image interoperability framework (iiif): A community \& technology approach for web-based images. In: Archiving Conference. vol. 2015, pp. 16-21. Society for Imaging Science and Technology (2015) 